\title{
ARCRAIDER II: Arc search in a sample of non-Abell clusters ${ }^{\star}$
}

\author{
W. Kausch ${ }^{1}$, S. Schindler ${ }^{1}$, T. Erben ${ }^{2}$, J. Wambsganss ${ }^{3}$, and A. Schwope ${ }^{4}$ \\ 1 Institut für Astrophysik, University of Innsbruck, Technikerstr. 25, 6020 Innsbruck, Austria \\ e-mail: wolfgang.kausch@uibk.ac.at \\ 2 Argelander-Institut für Astronomie (AIfA), University of Bonn, Auf dem Hügel 71, 53121 Bonn, Germany \\ 3 Astrophysikalisches Rechen-Institut und Universität Heidelberg, Mönchhofstr. 12-14, 16120 Heidelberg, Germany \\ 4 Astrophysikalisches Institut Potsdam (AIP), An der Sternwarte 16, 14482 Potsdam, Germany
}

Received 1 October 2008 / Accepted 6 January 2010

ABSTRACT

\begin{abstract}
Aims. We present a search for gravitational arcs in a unique sample of X-ray luminous, medium redshift clusters of galaxies. Methods. The sample of clusters is called ARCRAIDER, is based on the ROSAT Bright Survey (RBS) and fulfils the following criteria: (a) X-ray luminosity $\geq 0.5 \times 10^{45} \mathrm{erg} / \mathrm{s}(0.5-2 \mathrm{keV}$ band), (b) redshift range $0.1 \leq z \leq 0.52$, (c) classified as clusters in the RBS, (d) not a member of the Abell catalogue and, finally, (e) visible from the ESO sites La Silla/Paranal (declination $\delta \leq 20^{\circ}$ ). Results. In total we found more than 35 (giant) arc/arclet candidates, including a possible radial arc, one galaxy-galaxy lensing event and a possible quasar triple image in 14 of the 21 clusters of galaxies. Hence $66 \%$ of the sample members are possible lenses.
\end{abstract}

Key words. gravitational lensing: strong - cosmology: observation - galaxies: clusters: general

\section{Introduction}

Gravitational lensing techniques have become a blooming branch in astrophysics in the past with wide ranges of applications. In particular, the existence of strongly lensed objects offers the possibility to study the lenses, to investigate high redshift objects in more detail and can even be used for cosmological researches. Therefore systematic searches for gravitational arcs may provide an invaluable basis for those studies. Successful arc searches were already carried out by several authors, e.g. Bolton et al. (2008), Hennawi et al. (2008), Estrada et al. (2007), Sand et al. (2005), Luppino et al. (1999), Le Fevre et al. (1994), Smail et al. (1991) or Lynds \& Petrosian (1989).

A particular application for systematic arc searches is the statistical approach to arc frequencies, called arc statistics. Arc statistics investigate the probability of lensing events of specified properties. These probabilities depend on a large number of factors, e.g. the number density of sources and lenses, their properties, and the cosmological model.

Among the first to carry out arc statistic simulations in a cosmological context were Bartelmann et al. (1998, 2003, henceforth B98). They compared the frequency of arcs occurring in different cosmological models. Their main result was that the predicted number of arcs varies by orders of magnitudes between different cosmologies. In particular, the predicted frequency of arcs in the currently favoured $\Lambda$ CDM model is about one

* Based on observations made with ESO Telescopes at the La Silla or Paranal Observatories under programme IDs 60.A9123(G), 65.O-0425, 67.A-0444(A), 067.A-0095(B), 67.A-0427(A), 68.A-0255(A), 69.A-0010(A), 169.A-0595(G), 072.A-0083(A), and 073.A-0050(A). Also based on observations made with the NASA/ESA Hubble Space Telescope, and obtained from the Hubble Legacy Archive, which is a collaboration between the Space Telescope Science Institute (STScI/NASA), the Space Telescope European Coordinating Facility (ST-ECF/ESA) and the Canadian Astronomy Data Centre (CADC/NRC/CSA). order of magnitude too low compared to the estimated arc counts derived from observations. This led to lively discussions on the reasons for that discrepancy, as the $\Lambda$ CDM cosmology is widely supported by different observations, for example type I supernovae (Riess et al. 1998; Perlmutter et al. 1999), or cosmic microwave background observations (see e.g. Hanany et al. 2000; Pryke et al. 2002; Spergel et al. 2003). Therefore arc statistics simulations were refined by several authors: Flores et al. (2000) and Meneghetti et al. (2000) investigated with different methods whether contributions of individual cluster galaxies enlarge the cross section significantly. However, both found that cluster members do not increase the arc frequency significantly $(\lesssim 15 \%$, Flores et al. 2000). Additional effects of source ellipticities and sizes were investigated in detail by Keeton (2001), Oguri (2002) and Oguri et al. (2003).

The predictions on the lensing efficiency of simulated $0.2 \leq$ $z \leq 0.6$ clusters performed by Dalal et al. (2004) agree very well with observations based on the Einstein Medium Sensitivity Survey (henceforth EMSS, Luppino et al. 1999). They also found a strong dependency of about one order of magnitude of the cross section on the viewing angle of the cluster, which is caused by triaxiality and shallow density cusps of their simulated clusters.

While B98 assumed a constant source distance of $z_{\mathrm{s}}=1$ Wambsganss et al. (2004) showed that the lensing probability is a strong function of the source redshift, which was confirmed by Li et al. (2005). Varying the source redshift yields a much higher optical depth, hence the predicted arc frequency is significantly higher. Using only $z_{\mathrm{s}}=1$ sources Wambsganss et al. (2004) confirm the results of B98. Torri et al. (2004) investigated the influence of the dynamical state of galaxy clusters on arc statistics. They revealed that during merger processes the caustics change significantly, increasing the number of long and thin arcs by one order of magnitude. Another factor was introduced by Puchwein et al. (2005): they investigated the influence of the intracluster 

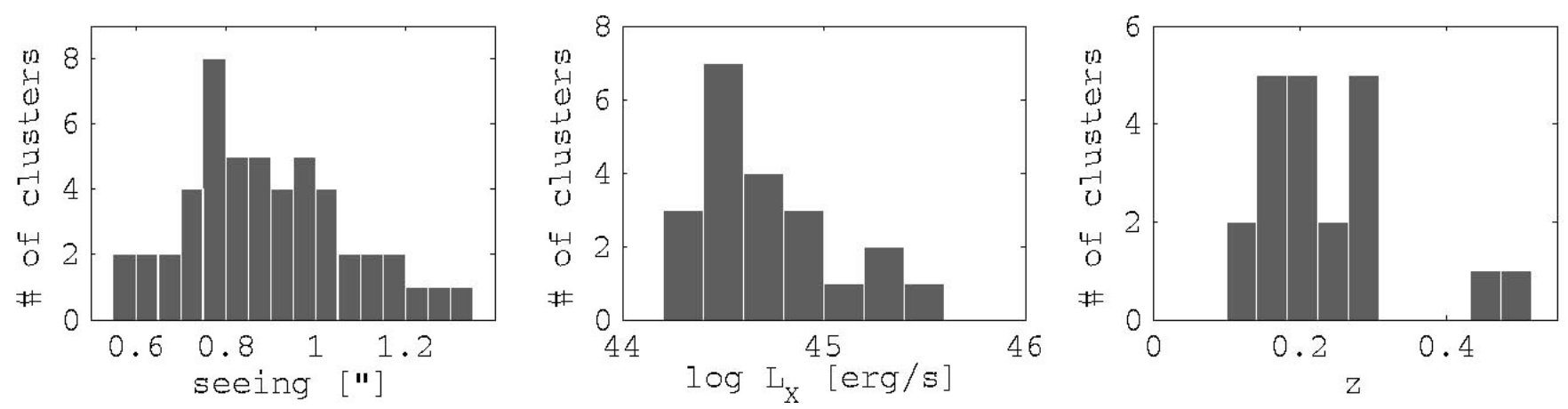

Fig. 1. Left: seeing histogram. As arc(lets) are often faint and thin structures, it is important to observe under good seeing conditions. The majority of our observations were performed with a seeing better than $1^{\prime \prime}$, the median value is 0. ' 87 . Middle: histogram of the $L_{X}$ distribution of the selected clusters. Right: redshift distribution of the sample members.

gas and its properties on the lensing efficiency and find a considerable impact under certain physical conditions. In particular, cooling and star formation processes may contribute to the lensing cross section by steepening the mass profile.

Numerical simulations nowadays show that the number of arcs for a $\Lambda \mathrm{CDM}$ cosmology roughly agrees with observations. However, a direct comparison is hardly possible as all simulations are based on idealised situations. In particular observers have to deal with observational effects like seeing, limiting magnitude, instrumental properties,... Which are not taken into account in the simulations at all. The reason is that those observational effects are not yet modelled properly, as they concern a wide range of different effects. For example, observations are usually based on a set of individual images, which are stacked on one single final frame. All images are unavoidably taken under slightly different conditions, hence the final frame contains a mixture of all individual image properties. The final effect of such a mixture is hard to judge. In particular, blurring due to different seeing conditions may affect the length-to-width ratio as well as the length of an arc, leading to different morphology detections. The first attempt taking observational effects into account was done by Horesh et al. (2005). They compared a sample of ten galaxy clusters based on HST observations (Smith et al. 2005) with simulations including some observational effects. Although the observed sample is very small and based on Abell clusters only, they found an agreement between arc frequency predictions for $\Lambda \mathrm{CDM}$ cosmology and the used observations.

We present a sample of galaxy clusters, which is particularly suited for arc statistics. In Sect. 2 we first describe the cluster sample in detail, its selection criteria, the observations and the data treatment, followed by a report on the methods used (Sect. 3). The results of the arc search are presented in Sect. 4, a summary and conclusion is given in Sect. 5. Throughout this paper we use a standard $\Lambda \mathrm{CDM}$ cosmology with $H_{0}=$ $70 h_{70}^{-1} \mathrm{~km} \mathrm{~s}^{-1} \mathrm{Mpc}^{-1}, \Omega_{\mathrm{M}}=0.3$, and $\Omega_{\Lambda}=0.7$.

\section{The ARCRAIDER project}

\subsection{Selection criteria}

ARCRAIDER stands for ARCstatistics with X-RAy lumInous meDium rEdhift galaxy clusteRs and is an ongoing long term project. It is based on the ROSAT Bright Survey (Schwope et al. 2000, henceforth RBS) and aimed at arc statistic studies. The RBS is a compilation of the brightest sources in the ROSAT All Sky Survey (Voges et al. 1999) with high galactic latitudes $\left(|b|>30^{\circ}\right)$ and a PSPC count rate of $>0.2 \mathrm{~s}^{-1}$. From this sample we selected objects fulfilling the criteria:

- classified as a cluster of galaxies;

- a redshift of $0.1 \leq z \leq 0.52$;

- X-ray luminosity $\log \left(L_{X}\right) \geq 0.5 \times 10^{45} \mathrm{erg} / \mathrm{s}(0.5-2 \mathrm{keV}$ band);

- visible from the ESO sites La Silla/Paranal (declination $\delta \leq$ $\left.20^{\circ}\right)$

- not a member of the Abell catalogue (Abell et al. 1989).

As the resulting 21 clusters (see Table 1 for more details) are located at high galactic latitudes, the $n_{\mathrm{H}}$ values are very small $\left(n_{\mathrm{H}} \leq 7.7 \times 10^{20} \mathrm{~cm}^{-2}\right)$. The members of the Abell catalogue were excluded at this first stage of the project as they are also frequent targets of observations during finished and ongoing studies. Hence it is not necessary to reobserve them which reduces our need for observing time.

\subsection{Observations}

The total number of the sample is 21 galaxy clusters, which were usually observed under good seeing condition (median seeing value 0.87", see Fig. 1) at different ESO telescopes (see Table 2 for more details on the used data, filters and proposalIDs). As the main instrument we chose SUSI2@NTT, except for RBS325, RBS653 and RBS864. These three clusters were observed with WFI@2.2 m, because we had to shift to this telescope during the scheduling process. All clusters were observed at least in the $V$ and the $R$ band to achieve colour information. Additional observations with different filters or instruments were used when available in the ESO archive (see Tables 2 and 3). The given limiting magnitude in Table 3 is defined as $m_{\text {lim }}=Z P-2.5 \log \left(\sqrt{N_{\text {pix }}} \cdot 3 \cdot \sigma\right)$, where $Z P$ is the magnitude zeropoint, $N_{\text {pix }}$ is the number of image pixels in a circle with a radius of 2'. 0 and $\sigma$ is the sky background noise. The used WFI filters were Ic/Iwp (ESO845), $R_{\mathrm{c}} / 162$ (ESO844), V/89 (ESO843), and, for RBS-0864, B/123 (ESO878), whereas B/99 (ESO842) was used for RBS-0325 and RBS-0653, respectively. The used SUSI2 Bessel filters were V\#812, R\#813, I\#814, the FORS filters U_BESS+33, B_BESS+34, V_BESS+35, R_BESS+36, and I BESS+37.

The data reduction was performed with the help of the GaBoDS pipeline. This software package was especially designed for multi-chip imagers and performs the basic reduction, superflatting and fringe correction, astrometric and photometric calibration and, finally, the coaddition. For more details on the used algorithms we refer the interested reader to Erben et al. (2005) and Erben \& Schirmer (2003). 
Table 1. Overview of the sample (see Schwope et al. 2000).

\begin{tabular}{|c|c|c|c|c|c|c|c|}
\hline $\begin{array}{c}\text { RBS } \\
\text { number }\end{array}$ & $\begin{array}{c}\alpha(\mathrm{J} 2000) \\
{[\mathrm{h} \mathrm{m} \mathrm{s}]}\end{array}$ & $\begin{array}{c}\delta(\mathrm{J} 2000) \\
{[\mathrm{d} \mathrm{m} \mathrm{s}]}\end{array}$ & $z$ & $1^{\prime \prime} 0=[\mathrm{kpc}]$ & $L_{\mathrm{X}}$ & $\begin{array}{c}n_{\mathrm{H}} \\
{\left[10^{22} \mathrm{e}^{-} / \mathrm{cm}^{2}\right]}\end{array}$ & $\begin{array}{l}\text { Alternative } \\
\text { name }\end{array}$ \\
\hline RBS-0172 & 011548.8 & -565529 & 0.272 & 4.16 & 44.8 & 3.39 & APMUKS(BJ) B011348.12-571116.8 \\
\hline RBS-0238 & 014511.9 & -603345 & 0.1795 & 3.03 & 44.6 & 3.36 & \\
\hline RBS-0312 & 022436.4 & -243344 & 0.305 & 4.51 & 44.8 & 1.69 & \\
\hline RBS- 0325 & 023216.4 & -442048 & 0.282 & 4.27 & 44.8 & 2.61 & \\
\hline RBS-0380 & 030107.5 & -470625 & 0.515 & 6.20 & 45.3 & 2.23 & \\
\hline RBS-0381 & 030138.5 & +015516 & 0.1690 & 2.88 & 44.5 & 7.7 & $\mathrm{ZwCl} 0258.9+0142, \mathrm{RHS} 18,4 \mathrm{C}+01.06$ \\
\hline RBS-0436 & 033106 & -210034 & 0.189 & 3.16 & 44.6 & 2.47 & \\
\hline RBS-0464 & 034254.2 & -370739 & 0.201 & 3.31 & 44.5 & 1.64 & \\
\hline RBS-0651 & 052815.1 & -294303 & 0.157 & 2.72 & 44.2 & 1.83 & [BCT2000] J052815.92-294300.8 \\
\hline RBS-0653 & 052852.7 & -392818 & 0.286 & 4.31 & 44.9 & 2.1 & NVSS J052853-392815 \\
\hline RBS-0745 & 090900.4 & +105935 & 0.1600 & 2.76 & 44.5 & 3.91 & MS 0906.3+1111 \\
\hline RBS-0864 & 102339.6 & +041110 & 0.2906 & 4.36 & 45.3 & 2.87 & ZwCl 1021.0+0426, Z3146 \\
\hline RBS-1015 & 114023.54 & +152810 & 0.24 & 3.79 & 44.9 & 2.65 & \\
\hline RBS-1029 & 114535.1 & -034002 & 0.1683 & 2.87 & 44.6 & 2.49 & SDSS J114535.10-034001.6 \\
\hline RBS-1267 & 132617.7 & 122958 & 0.2034 & 3.34 & 44.7 & 1.95 & [VCV2001] J132617.6+123000 \\
\hline RBS-1316 & 134732 & -114542 & 0.451 & 5.77 & 45.5 & 4.92 & RX J1347.5-1145, LCDCS 0829 \\
\hline RBS-1460 & 150407.6 & -024817 & 0.2169 & 3.51 & 45.2 & 5.98 & LCRS B150131.5-023636, QUEST J1504-0248 \\
\hline RBS-1691 & 204150.1 & -373339 & 0.100 & 1.84 & 44.2 & 3.61 & \\
\hline RBS-1712 & 210204.4 & -243358 & 0.1880 & 3.14 & 44.6 & 5.33 & EXO 2059-247 ID \\
\hline RBS-1748 & 212939.7 & +000518 & 0.233 & 3.71 & 45.0 & 4.22 & \\
\hline RBS-1842 & 221656.7 & -172527 & 0.1360 & 2.41 & 44.2 & 2.28 & [BCT2000] J221657.46-172528.3 \\
\hline
\end{tabular}

Table 2. Overview of the observations.

\begin{tabular}{|c|c|c|c|c|c|c|c|c|c|}
\hline $\begin{array}{c}\text { RBS } \\
\text { number }\end{array}$ & Instrument & $\begin{array}{l}\text { Binning } \\
\text { mode }\end{array}$ & $\begin{array}{c}U \\
t_{\exp }[\mathrm{s}]\end{array}$ & $\begin{array}{c}B \\
t_{\exp }[\mathrm{s}] \\
\end{array}$ & $\begin{array}{c}V \\
t_{\exp }[\mathrm{s}] \\
\end{array}$ & $\begin{array}{c}R \\
t_{\exp }[\mathrm{s}] \\
\end{array}$ & $\begin{array}{c}I \\
t_{\exp }[\mathrm{s}] \\
\end{array}$ & $\begin{array}{c}K \mathrm{~s} \\
t_{\mathrm{exp}}[\mathrm{s}] \\
\end{array}$ & $\begin{array}{l}\text { Proposal } \\
\text { IDs }\end{array}$ \\
\hline RBS-0172 & SUSI2@ESONTT & $2 \times 2$ & - & - & 3040 & 6080 & - & - & 67.A-0444(A) \\
\hline RBS-0238 & SUSI2@ESONTT & $2 \times 2$ & - & - & 3040 & 6080 & - & - & 67.A-0444(A) \\
\hline RBS-0312 & SUSI2@ESONTT & $2 \times 2$ & - & - & 3040 & 6080 & - & - & 67.A-0444(A) \\
\hline RBS-0325 & WFI@ESO2.2m & - & - & 5400 & 8000 & 16100 & - & - & 68.A-0255(A) \\
\hline RBS-0380 & SUSI2@ESONTT & $2 \times 2$ & - & - & 2250 & 2250 & - & - & 67.A-0444(A) \\
\hline RBS-0381 & SUSI2@ESONTT & $1 \times 1$ & - & - & 2600 & 5200 & - & - & 072.A-0083(A) \\
\hline \multirow[t]{2}{*}{ RBS-0436 } & SUSI2@ESONTT & $1 \times 1$ & - & - & 2600 & 5200 & - & - & 072.A-0083(A) \\
\hline & ACS@HST & - & - & - & $1200^{3}$ & - & - & - & 10881 \\
\hline RBS-0464 & SUSI2@ESONTT & $1 \times 1$ & - & - & 3600 & 5900 & - & - & 072.A-0083(A) \\
\hline \multirow[t]{2}{*}{ RBS-0651 } & SUSI2@ESONTT & $1 \times 1$ & - & - & 1740 & 5200 & - & - & 072.A-0083(A) \\
\hline & ACS@HST & - & - & - & $1200^{3}$ & - & - & - & 10881 \\
\hline RBS-0653 & WFI/ESO2.2m & - & - & 1200 & 8000 & 8000 & 1200 & - & 68.A-0255(A), 60.A-9123(G) \\
\hline RBS-0653 & VIMOS/IMG & - & - & - & - & 2400 & - & - & 169.A-0595(G) \\
\hline RBS-0745 & SUSI2@ESONTT & $2 \times 2$ & - & - & 3040 & 6080 & - & - & 69.A-0010(A) \\
\hline RBS-0864 ${ }^{a}$ & WFI@ESO2.2m & - & - & 1500 & 8000 & 25000 & - & - & 68.A-0255(A), 073.A-0050 \\
\hline RBS-1015 & SUSI2@ESONTT & $1 \times 1$ & - & - & 2600 & 5900 & - & - & 072.A-0083(A) \\
\hline RBS-1029 & SUSI2@ESONTT & $2 \times 2$ & - & - & 3040 & 6080 & - & - & 69.A-0010(A) \\
\hline RBS-1267 & SUSI2@ESONTT & $1 \times 1$ & - & - & 2200 & 6080 & - & - & 072.A-0083(A) \\
\hline RBS- $1316^{b}$ & FORS/ISAAC@VLT & High Res./- & 11310 & 4800 & 4500 & 6000 & 6750 & $\sim 7200$ & 67.A-0427(A), 067.A-0095(B) \\
\hline RBS-1460 & SUSI2@ESONTT & $2 \times 2$ & - & - & 3040 & 6080 & - & - & 69.A-0010(A) \\
\hline RBS-1691 & SUSI2@ESONTT & $2 \times 2$ & - & - & 3040 & 6080 & - & - & 69.A-0010(A) \\
\hline RBS-1712 & SUSI2@ESONTT & $2 \times 2$ & - & - & 15380 & 6080 & 9300 & - & 65.O-0425, 67.A-0444(A) \\
\hline \multirow[t]{2}{*}{ RBS-1748 } & SUSI2@ESONTT & $2 \times 2$ & - & - & 3040 & 6080 & - & - & 67.A-0444(A) \\
\hline & WFPC2@HST & - & - & - & $1200^{3}$ & - & - & - & 8301 \\
\hline RBS-1842 & SUSI2@ESONTT & $2 \times 2$ & - & - & 3800 & 7600 & - & - & 69.A-0010(A) \\
\hline
\end{tabular}

Notes. ${ }^{(a)}$ See Kausch et al. (2007), ${ }^{(b)}$ see Bradač et al. (2005) and Halkola et al. (2008).

The astrometric reference frame was tied to the USNO-A2 catalogue (Monet et al. 1998a,b), the photometric calibration was done with the STETSON standards (Stetson 2000). All magnitudes are given in the Vega system.

For nights where no standard star observations were observed we took the standard zero points given on the SUSI2 homepage $^{1}$ after investigating the photometric conditions of these nights with the help of WFI observations.

${ }^{1}$ http://www.ls.eso.org/lasilla/sciops/ntt/susi/docs/ SUSIphot.html
Where available we also used HST data taken from the archive. This concerns the clusters RBS-0436 a RBS-0651 ${ }^{\mathrm{a}}$, RBS- $0864^{\mathrm{b}}$ and RBS-1748 ${ }^{\mathrm{b}}$, which were observed during snapshot programmes (filter: F606W; ${ }^{a}$ PID: 10881, P.I. Smith, ACS, $t_{\exp }=1200 \mathrm{~s}$; ${ }^{b}$ PID: 8301, P.I.: Edge, WFPC2, $\left.t_{\exp }=1000 \mathrm{~s}\right)$. We used the calibrated images from the Hubble Legacy Archive ${ }^{2}$ mainly to identify possible arcs, as those images are well-suited

\footnotetext{
2 http://hla.stsci.edu/
} 
Table 3. Table with measured limiting magnitude values.

\begin{tabular}{ccccc}
\hline \hline $\begin{array}{c}\text { RBS } \\
\text { cluster }\end{array}$ & $\begin{array}{c}m_{\lim } \\
\text { in } B[\mathrm{mag}]\end{array}$ & $\begin{array}{c}m_{\lim } \\
\text { in } V[\mathrm{mag}]\end{array}$ & $\begin{array}{c}m_{\lim } \\
\text { in } R[\mathrm{mag}]\end{array}$ & $\begin{array}{c}m_{\lim } \\
\text { in } I[\mathrm{mag}]\end{array}$ \\
\hline RBS-0172 & - & 25.67 & 25.73 & - \\
RBS-0238 & - & 25.63 & 25.57 & - \\
RBS-0312 & - & 25.66 & 25.55 & - \\
RBS-0325 & 25.81 & 25.46 & 25.49 & - \\
RBS-0380 & - & 25.22 & 25.44 & - \\
RBS-0381 & - & 25.95 & 25.74 & - \\
RBS-0436 & - & 25.71 & 25.29 & - \\
RBS-0464 & - & 25.72 & 25.69 & - \\
RBS-0651 & - & 25.27 & 25.80 & - \\
RBS-0653 & 24.89 & 25.07 & 25.76 & 22.35 \\
RBS-0745 & - & 25.13 & 25.18 & - \\
RBS-1015 & - & 26.19 & 25.80 & - \\
RBS-1029 & - & 25.30 & 25.21 & - \\
RBS-1267 & - & 25.86 & 24.40 & - \\
RBS-1460 & - & 25.12 & 25.34 & - \\
RBS-1691 & - & 25.41 & 25.48 & - \\
RBS-1712 & - & 25.19 & 25.91 & 24.70 \\
RBS-1748 & - & 25.51 & 25.47 & - \\
RBS-1842 & - & 25.71 & 25.55 & - \\
\hline
\end{tabular}

due to their missing atmospheric blurring, even in spite of the very short exposure times.

\section{Methods}

\subsection{Determination and photometry of the arc candidates}

One of the main issues of the ARCRAIDER project at this stage is the search for gravitational arcs. As arcs are difficult to detect, we have to focus on the most important criteria. In ground-based observations usually only arcs tangentially aligned with respect to the mass centre are visible. Radial arcs are often too thin and too faint structures in the vicinity of bright central galaxies of clusters. In addition, arcs and their counter images have the same spectra and redshifts of the order of $\gtrsim 2 \times z_{\text {lens }}$. However, as we do not have spectra, we restrict our search criteria to the morphology, the position and alignment of the possible candidates with respect to a bright central cluster galaxy (BCG), assuming the latter to be the centre.

To determine the length-to-width ratio $l / w$ to be used as selection criterion we follow an ansatz by Lenzen et al. (2004) and the SExtractor Manual (Bertin 2005) and define the $l / w$ ratio by calculating the eigenvalues $\lambda_{1}$ and $\lambda_{2}$ of the second order moment of the light distribution $L_{k l}$ (note that $L_{12}=L_{21}$ ):

$$
\begin{aligned}
& \lambda_{1}^{2}=\frac{L_{11}+L_{22}}{2}+\sqrt{\left(\frac{L_{11}-L_{22}}{2}\right)^{2}+L_{12}} \\
& \lambda_{2}^{2}=\frac{L_{11}+L_{22}}{2}-\sqrt{\left(\frac{L_{11}-L_{22}}{2}\right)^{2}+L_{12} .}
\end{aligned}
$$

Although neither the length $l$ nor the width $w$ are equal to their counterparts $\lambda_{1}$ and $\lambda_{2}$, the ratio $l / w$ is equal to $\lambda_{1} / \lambda_{2}$ (Jähne 2002). Hence we obtain the length-to-width ratio by determining $\lambda_{1}$ and $\lambda_{2}$.

Though a lot of useful information (e.g. length-to-width ratio, location/orientation with respect to the BCG...) is contained in the catalogues, it is insufficient to restrict the search to object catalogues only. In several cases arcs merge apparently with foreground objects and can therefore be missed or are simply too faint to be detected. Hence an arc search is best performed by visual inspection of deep images and not restricted to catalogues

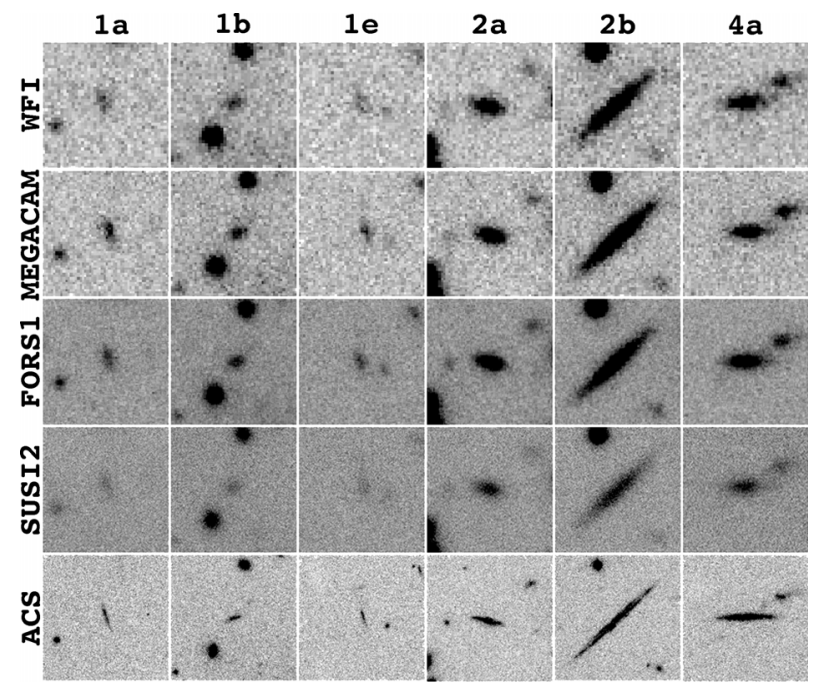

Fig. 2. Comparison of various gravitational arcs seen with five different imagers: WFI@ESO2.2m (filter $R_{\mathrm{c}} 162$ ), MEGACAM@CFHT (SDSS-r), FORS1@VLT (R_BESS+36), SUSI2@NTT (WB655\#825) and ACS $(F 814 W)$ onboard the HST. The naming of the arcs is taken from Halkola et al. (2008).

only. We use the following selection criteria for arc candidates: (a) they are tangentially aligned, and, (b) in a distance of $<1^{\prime}$, both with respect to the central cluster galaxy, and (c) show a length-to-width ratio of $l / w \geq 1.5$ (measured with SExtractor, see Sect. 3.2 for more details).

We additionally assigned the arc candidates to two classes (A, and B). Class A denotes a high probability of being a lensed object, whereas B type objects are of low probability, but not excludable lensing features. This separation is primarily meant to be as priority list for subsequent observations.

All magnitudes were determined with SExtractor in double image mode using MAG_AUTO with the following parameters for all clusters: DETECT_MINAREA $=3$, effective GAIN $=t_{\exp } * G_{\text {AIN }}$ INSTR. The GAIN INSTR is 2 for WFI and 2.25 for SUSI2. We also took galactic extinction $E(B-V)$ into account based on values by Schlegel et al. (1998), in spite of the low values due to the high galactic latitude bias introduced by the RBS catalogue. The separation of stars and galaxies was done using CLASS_STAR $<0.95$.

Additional information about the arc candidates can be derived from their colour information. We have compared the $(V-R)$ colours of the arcs with the average colour of the five brightest cluster members (see Table 9). As lensed objects are highly redshifted galaxies of various types, their colour usually differs from the main lensing cluster members. Except for three of them (RBS-0238: B2; RBS-0651: B3; RBS-1460: B1) all candidates show different colours than the five brightest cluster members, which indicates their non-cluster membership.

\subsection{Morphology of the arc candidates/mass estimates}

At first glance the chosen limit of the length-to-width ratio $l / w \geq 1.5$ for arc determination seems to be very low. The reason is the atmospheric blurring, which dramatically decreases the $l / w$. Figure 2 shows $R$-band images of five gravitational arclets and three arcs discovered in RXJ1347-1145 (see Bradač et al. 2005; Halkola et al. 2008) as seen by four different ground based imagers (WFI@ESO2.2m, MEGACAM@CFHT, FORS1@ESOVLT, SUSI2@ESONTT) in comparison with $F 814 W$ frame observations (no $R$ band was available) taken 
Table 4. Technical data of the images in Fig. 2.

\begin{tabular}{ccccc}
\hline \hline Imager & $\begin{array}{c}\text { Pixel } \\
\text { scale }\end{array}$ & $\begin{array}{c}\text { Measured } \\
\text { seeing ["] }\end{array}$ & $\begin{array}{c}\text { Exposure } \\
\text { time }[\mathrm{s}]\end{array}$ & $\begin{array}{c}m_{\mathrm{lim}} \\
{[\mathrm{mag}]}\end{array}$ \\
\hline WFI & 0.238 & 0.85 & 16300 & 25.4 \\
MEGACAM & 0.186 & 0.75 & 7200 & 25.9 \\
FORS1 & 0.1 & 0.69 & 6000 & 26.5 \\
SUSI2 & 0.0805 & 0.91 & 11250 & 25.9 \\
ACS & 0.05 & n/a & 5280 & 26.45 \\
\hline
\end{tabular}

Table 5. Measured $l / w$ of the arcs shown in Fig. 2.

\begin{tabular}{ccccccc}
\hline \hline Imager & $1 \mathrm{a}$ & $1 \mathrm{~b}$ & $1 \mathrm{e}$ & $2 \mathrm{a}$ & $2 \mathrm{~b}$ & $4 \mathrm{a}$ \\
\hline WFI & 1.6 & 1.5 & 1.1 & 1.7 & 4.5 & 2.5 \\
MEGACAM & 2.0 & 1.5 & 1.9 & 1.8 & 5.0 & 2.5 \\
FORS1 & 1.8 & 1.4 & 1.7 & 1.5 & 4.9 & 2.6 \\
SUSI2 & 1.8 & 1.3 & 1.4 & 1.7 & 4.7 & 2.4 \\
ACS & 2.6 & 3.1 & 3.8 & 4.3 & 8.1 & 7.4 \\
BF & 0.69 & 0.46 & 0.40 & 0.39 & 0.59 & 0.34 \\
\hline
\end{tabular}

with the ACS onboard the HST (see Table 4). All observations are very deep $\left(\mathrm{mag}_{\lim } \leq 25.4\right)$ and all ground based images were taken under excellent seeing conditions (seeing $<1^{\prime \prime}$ ). To estimate the influence of $l / w$ measurements caused by atmospheric blurring we define the blurring factor $\mathrm{BF}$ as the ratio $(l / w)_{\mathrm{gba}} /(l / w)_{\mathrm{ACS}}$, (where gba stands for "ground based average").

Except for object 1e, which is by far the smallest and faintest, the length-to-width ratio measurements on the ground based images roughly agrees (see Table 5). However, it is clearly visible that the $l / w$ is dramatically higher in the HST frame, which is shown by a blurring factor BF of down to $\sim 0.4$. This means that the influence of atmospheric blurring is highly dominant over other factors like different spatial sampling (pixel scale), or exposure times. Hence the fraction of missed arc candidates is minimized with an assumed limit of the ground based lengthto-width ratio $l / w \geq 1.5$ for arc determination. However, the contamination by cluster or foreground elongated objects is increased. Thus a spectroscopic confirmation of the arc candidates is mandatory in the future.

We can also use the position of the arcs to roughly estimate the mass of the lensing galaxy assuming it is a part of an Einstein ring. As we do not know the distance to the background galaxy we only get a rough estimate of the mass within the Einstein ring by assuming $z_{\text {background }}=1$, the upper/lower limits are estimated by assuming $z_{\text {background }}=\left[2 \times z_{\text {cluster }}, 2\right]$. Due to the large number of assumptions we concentrate on A class candidates only.

\section{Results and discussion}

\subsection{Individual clusters}

The ARCRAIDER sample includes several clusters with distinct arc like features (see the Appendix for the images). Table 9 shows a list of all measurable photometric and morphologic quantities of the arc candidates. They are usually named by their classification (see Sect. 3.1) followed by a running number. The distances are measured from the cluster centres (hereafter "cc"), the given masses are assumed as being masses within an Einstein radius of the $\mathrm{A}$ class candidates with a source redshift $z_{s}=1$, the errors are calculated using $z_{s}=2 * z_{\text {cluster }}, 2$. As some arcs are very faint structures it was not possible to obtain reliable photometric information or morphologic estimates (marked by “-”).
Table 6. Sample divided into three classes I, II, and III, with respect to the X-ray luminosity $L_{\mathrm{X}}$.

\begin{tabular}{llcc}
\hline \hline $\begin{array}{l}L_{\mathrm{X}} \\
\text { class }\end{array}$ & $\begin{array}{c}\# \\
\text { clusters }\end{array}$ & $\begin{array}{c}\text { \# of A-type arc } \\
\text { cand. per cluster }\end{array}$ \\
\hline I & {$\left[\log \left(L_{\mathrm{X}}\right) \leq 44.5\right]$} & 6 & 0.33 \\
II & {$\left[44.5<\log \left(L_{\mathrm{X}}\right)<44.9\right]$} & 8 & 0.5 \\
III & {$\left[44.9 \leq \log \left(L_{\mathrm{X}}\right)\right]$} & 7 & 3.14 \\
\hline
\end{tabular}

Table 7. The three X-ray luminosity classes of the EMSS cluster sample. See text for more details.

\begin{tabular}{llc}
\hline \hline$L_{\mathrm{X}}[0.5-2 \mathrm{keV}]$ & & $\#$ \\
EMSS sample class & & clusters \\
\hline I & {$\left[\log \left(L_{\mathrm{X}}\right) \leq 44.32\right]$} & 11 \\
II & {$\left[44.32<\log \left(L_{\mathrm{X}}\right)<44.47\right]$} & 10 \\
III & {$\left[44.47 \leq \log \left(L_{\mathrm{X}}\right)\right]$} & 12 \\
\hline
\end{tabular}

Table 8. Mean values of the X-ray luminosity classes (see Tables 7 and 6 , respectively).

\begin{tabular}{ccc}
\hline \hline $\begin{array}{c}L_{\mathrm{X}} \\
\text { class }\end{array}$ & $\begin{array}{c}\text { EMSS sample } \\
\times 10^{45} \mathrm{erg} / \mathrm{s}\end{array}$ & $\begin{array}{c}\text { RBS sample } \\
\times 10^{45} \mathrm{erg} / \mathrm{s}\end{array}$ \\
\hline I & $\left\langle L_{\mathrm{X}}\right\rangle=0.16$ & $\left\langle L_{\mathrm{X}}\right\rangle=0.24$ \\
II & $\left\langle L_{X}\right\rangle=0.26$ & $\left\langle L_{\mathrm{X}}\right\rangle=0.50$ \\
III & $\left\langle L_{\mathrm{X}}\right\rangle=0.49$ & $\left\langle L_{\mathrm{X}}\right\rangle=1.62$ \\
\hline
\end{tabular}

In particular RBS-0325, RBS-0651, RBS-0653, RBS-0864 (Kausch et al. 2007) and RBS-1316 (RXJ1347-1145, see e.g. Bradač et al. 2005; Halkola et al. 2008) show features, which are very probably lensed objects. Apart from that several small arclet candidates, the B-typed objects, can be found in various clusters of the sample, but it is unlikely that they are lenses. In addition we found a galaxy-galaxy lensing candidate in RBS-0312, a candidate for a radial arc in RBS-0325 (see Figs. A.1c and d, respectively), and a possible multi-imaged quasar in RBS-1712 (Fig. A.3c).

\subsection{Correlation of the $X$-ray luminosity and the number of arc candidates}

A correlation between the X-ray luminosity and the number of arcs is expected, because the X-ray luminosity correlates with the mass of the cluster (Schindler 1999; Reiprich \& Böhringer 1999) and the probability to detect arcs increases with the cluster mass. Dividing the sample into classes with the X-ray luminosity intervals I: $\left[44.2 \leq \log \left(L_{X}\right) \leq 44.5\right]$, II: $\left[44.5<\log \left(L_{X}\right)<\right.$ 44.9], and III: [44.9 $\left.\leq \log \left(L_{X}\right)\right]$ to obtain three classes of approximately similar size, we find a strong correlation between the number of A-type arc candidates and the X-ray luminosity in the preliminary sample with 0.33 arc candidates per cluster in the faintest class and 3.14 arc candidates per cluster in the most luminous bin (see Table 6).

Seven of the 14 ARCRAIDER lensing cluster candidates show B-type objects only, and six at least one class A type. Additionally $\sim 10 \%$ are really impressive lensing clusters (RBS-0653 and RBS-1316), containing a large number of candidates and giant arcs.

\subsection{Comparison with the EMSS sample}

Several other arc searches have been carried out by various groups (e.g. Bolton et al. 2008; Hennawi et al. 2008; Estrada et al. 2007; Sand et al. 2005). However, a direct comparison with 
A\&A 513, A8 (2010)

Table 9. List of the photometric and morphologic quantities of measurable arcs and arc candidates, except for RBS-0864 (see Kausch et al. 2007) and RBS1316 (RXJ1347-1145, see Bradač et al. 2005). See text for more details.

\begin{tabular}{|c|c|c|c|c|c|c|c|c|c|c|c|}
\hline Cluster & Arc & $\begin{array}{c}I \\
{[\mathrm{mag}]}\end{array}$ & $\begin{array}{c}R \\
{[\mathrm{mag}]}\end{array}$ & $\begin{array}{c}V \\
{[\mathrm{mag}]}\end{array}$ & $\begin{array}{c}B \\
{[\mathrm{mag}]}\end{array}$ & $\begin{array}{c}(V-R)\rangle \\
\text { cluster }^{g}\end{array}$ & $\begin{array}{c}(V-R) \\
\operatorname{arcs} \\
\end{array}$ & $\overline{l l / w}$ & $\begin{array}{l}\text { Distance to } \\
\mathrm{cc}\left[{ }^{\prime \prime}\right]\end{array}$ & $\begin{array}{l}\text { Distance to } \\
\text { cc }\left[h_{70}^{-1} \mathrm{kpc}\right]\end{array}$ & $\begin{array}{c}\text { Mass } \\
{\left[\times 10^{14} M_{\odot}\right]}\end{array}$ \\
\hline RBS-0172 & B1 & - & $23.28 \pm 0.02$ & $24.16 \pm 0.07$ & - & 0.72 & $0.88 \pm 0.09$ & 1.5 & $\sim 29$ & $\sim 120$ & - \\
\hline \multirow[t]{3}{*}{ RBS-0238 } & B1 & - & $22.42 \pm 0.04$ & $23.31 \pm 0.05$ & - & 0.72 & $0.89 \pm 0.10$ & 2.5 & $\sim 20$ & $\sim 60$ & - \\
\hline & $\mathrm{B} 2^{a}$ & - & $23.61 \pm 0.07$ & $24.32 \pm 0.08$ & - & 0.72 & $0.70 \pm 0.15$ & 2.0 & $\sim 50$ & $\sim 150$ & - \\
\hline & $\mathrm{B}^{a}{ }^{a}$ & - & - & - & - & - & - & - & $\sim 20$ & $\sim 60$ & - \\
\hline RBS-0312 & $\mathrm{GGL}^{b}$ & - & - & - & - & - & - & - & $\sim 2.4$ & $\sim 11$ & $0.0104_{-0.002}^{+0.004}$ \\
\hline \multirow[t]{4}{*}{ RBS-0325 } & A1 & - & $21.02 \pm 0.01$ & $21.48 \pm 0.02$ & $21.79 \pm 0.03$ & 0.84 & $0.47 \pm 0.02$ & 1.7 & $\sim 50$ & $\sim 210$ & $4.04_{-0.64}^{+1.73}$ \\
\hline & B2 & - & $22.58 \pm 0.01$ & $23.38 \pm 0.07$ & $24.09 \pm 0.23$ & 0.84 & $1.80 \pm 0.08$ & 1.7 & $\sim 47$ & 200 & -0.04 \\
\hline & A3 & - & $22.89 \pm 0.03$ & $24.29 \pm 0.12$ & $24.60 \pm 0.30$ & 0.84 & $1.40 \pm 0.15$ & 2.6 & $\sim 30$ & $\sim 130$ & $1.48_{-0.23}^{+0.63}$ \\
\hline & B4 & - & - & - & - & - & - & - & $\sim 38$ & $\sim 162$ & $2.38_{-0.37}^{+1.01}$ \\
\hline RBS-0436 & B1 & - & $23.04 \pm 0.05$ & $23.47 \pm 0.01$ & - & 0.56 & $0.42 \pm 0.06$ & 2.7 & $\sim 41$ & $\sim 130$ & - \\
\hline \multirow[t]{3}{*}{ RBS-0651 } & A1 & - & $21.11 \pm 0.01$ & $22.31 \pm 0.05$ & - & 0.59 & $1.20 \pm 0.05$ & 2.5 & $\sim 25$ & $\sim 67$ & $0.52_{-0.04}^{+0.35}$ \\
\hline & A2 & - & - & - & - & - & - & - & $\sim 12$ & $\sim 30$ & $\left.0.123_{-0.01}^{+0.08}\right)^{f}$ \\
\hline & B3 & - & $21.38 \pm 0.01$ & $22.79 \pm 0.09$ & - & 0.59 & $0.58 \pm 0.20$ & 2.8 & $\sim 37$ & $\sim 100$ & - \\
\hline \multirow[t]{4}{*}{ RBS-0653 } & $\mathrm{GA} 1^{d}$ & - & - & - & - & - & - & - & $\sim 20$ & $\sim 87$ & $6.67_{-1.07}^{+2.80}$ \\
\hline & GA2 & - & - & - & - & - & - & - & $\sim 11$ & $\sim 47$ & $2.02_{-0.32}^{+0.85}$ \\
\hline & GA3 & - & - & - & - & - & - & - & $\sim 19$ & $\sim 82$ & $6.02_{-0.97}^{+2.53}$ \\
\hline & A3 & $18.12 \pm 0.01$ & $19.23 \pm 0.01$ & $20.28 \pm 0.01$ & $21.38 \pm 0.09$ & 1.93 & $1.05 \pm 0.08$ & 2.1 & $\sim 12$ & $\sim 52$ & $0.25_{-0.04}^{+0.10}$ \\
\hline RBS-1015 & B1 & - & $23.79 \pm 0.04$ & $24.62 \pm 0.02$ & - & 0.66 & $0.83 \pm 0.06$ & 1.9 & $\sim 38$ & $\sim 145$ & - \\
\hline RBS-1029 & B1 & - & $22.36 \pm 0.03$ & $23.12 \pm 0.03$ & - & 0.59 & $0.76 \pm 0.06$ & 1.9 & $\sim 37$ & $\sim 105$ & - \\
\hline RBS-1460 & B1 & - & $23.39 \pm 0.07$ & $24.06 \pm 0.07$ & - & 0.65 & $0.67 \pm 0.15$ & 2.2 & $\sim 42$ & $\sim 148$ & - \\
\hline \multirow[t]{2}{*}{ RBS-1712 } & A1 & $22.66 \pm 0.05$ & - & $23.29 \pm 0.02$ & - & - & - & 2.6 & $\sim 20$ & $\sim 63$ & $0.41_{-0.04}^{+0.25}$ \\
\hline & $\mathrm{A} 2$ & $23.34 \pm 0.05$ & $24.17 \pm 0.13$ & $23.70 \pm 0.02$ & - & 0.64 & $-0.47 \pm 0.15$ & 1.9 & $\sim 21$ & $\sim 66$ & $0.45_{-0.45}^{+0.28}$ \\
\hline \multirow[t]{2}{*}{ RBS-1748 } & $\mathrm{B} 1^{e}$ & - & $25.09 \pm 0.05$ & $25.27 \pm 0.14$ & - & 0.57 & $0.18 \pm 0.18$ & $2.2^{5}$ & $\sim 31$ & $\sim 115$ & - \\
\hline & B2 & - & $24.78 \pm 0.04$ & $25.11 \pm 0.14$ & - & 0.57 & $0.33 \pm 0.18$ & $1.9^{5}$ & $\sim 35$ & $\sim 130$ & - \\
\hline
\end{tabular}

Notes. ${ }^{(a)}$ merging with another object; ${ }^{(b)}$ galaxy-galaxy lensing candidate; ${ }^{(c)}$ mass of the single galaxy with GGL1; ${ }^{(d)} \mathrm{GA}=$ giant arc; ${ }^{(e)}$ measured on the HST frame; ${ }^{(f)}$ mass of the BCG; ${ }^{(g)}$ averaged colour of the five brightest cluster galaxies.

those studies is hampered by considerable differences between the studies e.g. in the selection criteria of the cluster samples, photometric depth, or chosen instruments.

The only comparable search for gravitational arcs in clusters of galaxies was performed by Luppino et al. (1999), who searched for strong lensing features in 38 X-ray selected clusters taken from the EMSS (Gioia et al. 1990; Stocke et al. 1991). In total they discovered 16 clusters with arcs and arc candidates, including eight systems with giant arcs. $60 \%$ of their clusters exceeding $L_{\mathrm{X}}>10^{45} \mathrm{erg} \mathrm{s}^{-1}(0.3-3.5 \mathrm{keV}$ band) inhabitate giant arcs and none of the 15 clusters with $L_{X}<4 \times 10^{44} \mathrm{erg} \mathrm{s}^{-1}$ (same band) shows any strong lensing feature candidate.

Due to the similarities in the samples we can roughly compare the fraction of clusters inhabitating gravitational arcs between both samples. Because of the uncertainties in the arc determination we only take clusters into account, which show arcs, which are very probably strong lensing features. Hence, we use only type A arcs for our RBS sample (see Sect.3.2), for the EMSS sample we use giant arcs and arcs without a question mark in the last column of Table 1 in Luppino et al. (1999) and additionally only select clusters with a comparable redshift $(z \leq 0.515)$.

For this comparison we also divided the EMSS sample members into three classes of similar number counts with respect to the X-ray luminosity (see Table 7), which was transferred to the 0.5-2.0 keV band with the online PIMMS-Tool ${ }^{3}$.

Figure 3 shows a comparison of the lensing cluster fraction in the EMSS and the RBS sample, respectively. The dots mark the mean value of the X-ray luminosity of the clusters within the class (see Tables 6 and 7) of the corresponding sample (see

3 http://heasarc.nasa.gov/Tools/w3pimms.html

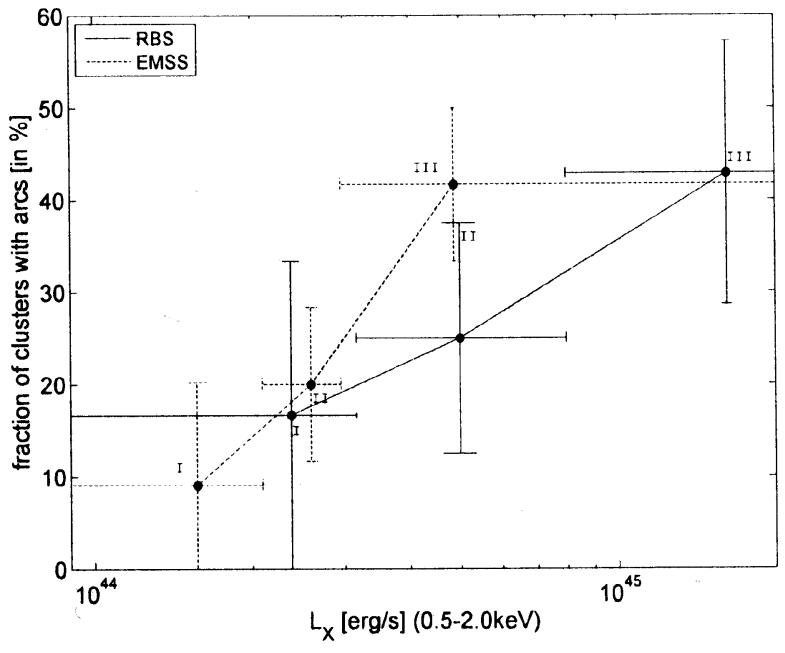

Fig. 3. Comparison of the lensing cluster fractions in the EMSS sample (dashed line) (Luppino et al. 1999) and the RBS sample (solid line). For both samples we used only the most secure arc candidates (see Sect. 4.3). The dots mark the mean values of the X-ray luminosity within the corresponding $L_{\mathrm{X}}$ classes I, II, and III, respectively (see Table 8), error bars in $L_{X}$ mark the limits given in Table 6. The error bars in the $y$-direction are assumed to be \pm 1 cluster with arcs. See Sect. 4.3 for more detail.

Table 8). The error bars in $x$-direction denote the limits of the corresponing $L_{X}$ class, in the $y$-direction the errors are assumed to be \pm 1 cluster with missed or misinterpreted arcs, respectively.

Surprisingly, the agreement between the samples is not as good as one one would expect for two similar samples. Both are strictly selected by $L_{X}$, except that the luminosity cut is much 
higher in the RBS sample. However, the discrepancy between the two samples could be caused by the momentary skipping of the Abell clusters ( $\sim 3 \%$ of the clusters in class I, $\sim 63 \%$ in class II, and $\sim 82 \%$ in class III). Although the Abell clusters are selected by visible light luminosity only, the omitting excludes several famous prominent lensing clusters (Abell 2204, Abell 2667, Abell 1835, Abell 1689, see e.g. Sand et al. 2005; Broadhurst et al. 2005).

\section{Summary}

We present a systematic search for gravitational arcs in a unique sample of X-ray-luminous, medium redshifted galaxy clusters. The search is based on deep ground based images taken with ESO telescopes under good seeing conditions (usually $<1^{\prime \prime}$ ). Including RBS-0864 (Z3146, Kausch et al. 2007) and RBS-1316 (RXJ1347-1145, Bradač et al. 2005; Halkola et al. 2008), respectively, the sample consists of 21 members. In total we found candidates for more than 35 (giant) arcs or arclets, one radial arc candidate, one galaxy-galaxy lensing event and three possible quasar lensing features in 14 sample members. Hence $66 \%$ of the clusters are possible strong lenses, with a strong bias towards X-ray luminous clusters (see Sect. 4.2).

The next step in the ARCRAIDER project is the analysis of the currently excluded Abell clusters belonging to this sample and spectroscopic follow-up observations of the arc candidates to confirm their lensing nature. Including these missing clusters and observations the ARCRAIDER sample is by far the largest for future arc statistic studies.

Acknowledgements. This work is supported by the Austrian Science Foundation (FWF) project number 15868.

\section{References}

Abell, G. O., Corwin, H. G., \& Olowin, R. P. 1989, ApJS, 70, 1

Bartelmann, M., Huss, A., Colberg, J. M., Jenkins, A., \& Pearce, F. R. 1998, A\&A, 330, 1

Bartelmann, M., Meneghetti, M., Perrotta, F., Baccigalupi, C., \& Moscardini, L. 2003, A\&A, 409, 449

Bertin, E. 2005, SExtractor v2.4 User's Manual
Bolton, A. S., Burles, S., Koopmans, L. V. E., et al. 2008, ApJ, 682, 964 Bradač, M., Erben, T., Schneider, P., et al. 2005, A\&A, 437, 49 Broadhurst, T., Benítez, N., Coe, D., et al. 2005, ApJ, 621, 53 Dalal, N., Holder, G., \& Hennawi, J. F. 2004, ApJ, 609, 50

Erben, T., \& Schirmer, M. 2003, GaBoDS Pipeline Documentation Ver. 0.5 Erben, T., Schirmer, M., Dietrich, J. P., et al. 2005, Astron. Nachr., 326, 432 Estrada, J., Annis, J., Diehl, H. T., et al. 2007, ApJ, 660, 1176 Flores, R. A., Maller, A. H., \& Primack, J. R. 2000, ApJ, 535, 555 Gioia, I. M., Maccacaro, T., Schild, R. E., et al. 1990, ApJS, 72, 567 Halkola, A., Hildebrandt, H., Schrabback, T., et al. 2008, A\&A, 481, 65 Hanany, S., Ade, P., Balbi, A., et al. 2000, ApJ, 545, L5

Hennawi, J. F., Gladders, M. D., Oguri, M., et al. 2008, AJ, 135, 664

Horesh, A., Ofek, E. O., Maoz, D., et al. 2005, ApJ, 633, 768

Jähne, B. 2002, Digitale Bildverarbeitung, 5th edn. (Springer)

Kausch, W., Gitti, M., Erben, T., \& Schindler, S. 2007, A\&A, 471, 31

Keeton, C. R. 2001, ApJ, 562, 160

Le Fevre, O., Hammer, F., Angonin, M. C., Gioia, I. M., \& Luppino, G. A. 1994, ApJ, 422, L5

Lenzen, F., Schindler, S., \& Scherzer, O. 2004, A\&A, 416, 391

Li, G.-L., Mao, S., Jing, Y. P., et al. 2005, ApJ, 635, 795

Luppino, G. A., Gioia, I. M., Hammer, F., Le Fèvre, O., \& Annis, J. A. 1999, A\&AS, 136, 117

Lynds, R., \& Petrosian, V. 1989, ApJ, 336, 1

Meneghetti, M., Bolzonella, M., Bartelmann, M., Moscardini, L., \& Tormen, G. 2000, MNRAS, 314, 338

Monet, D. B. A., Canzian, B., \& Dahn, C. 1998a, VizieR Online Data Catalog, 1252,0

Monet, D. B. A., Canzian, B., Dahn, C., et al. 1998b, VizieR Online Data Catalog, 1252, 0

Oguri, M. 2002, ApJ, 573, 51

Oguri, M., Lee, J., \& Suto, Y. 2003, ApJ, 599, 7

Perlmutter, S., Aldering, G., Goldhaber, G., et al. 1999, ApJ, 517, 565

Pryke, C., Halverson, N. W., Leitch, E. M., et al. 2002, ApJ, 568, 46

Puchwein, E., Bartelmann, M., Dolag, K., \& Meneghetti, M. 2005, A\&A, 442, 405

Reiprich, T. H., \& Böhringer, H. 1999, Astron. Nachr., 320, 296

Riess, A. G., Filippenko, A. V., Challis, P., et al. 1998, AJ, 116, 1009

Sand, D. J., Treu, T., Ellis, R. S., \& Smith, G. P. 2005, ApJ, 627, 32

Schindler, S. 1999, A\&A, 349, 435

Schlegel, D. J., Finkbeiner, D. P., \& Davis, M. 1998, ApJ, 500, 525

Schwope, A., Hasinger, G., Lehmann, I., et al. 2000, Astron. Nachr., 321, 1

Smail, I., Ellis, R. S., Fitchett, M. J., et al. 1991, MNRAS, 252, 19

Smith, G. P., Kneib, J.-P., Smail, I., et al. 2005, MNRAS, 359, 417

Spergel, D. N., Verde, L., Peiris, H. V., et al. 2003, ApJS, 148, 175

Stetson, P. B. 2000, PASP, 112, 925

Stocke, J. T., Morris, S. L., Gioia, I. M., et al. 1991, ApJS, 76, 813

Torri, E., Meneghetti, M., Bartelmann, M., et al. 2004, MNRAS, 349, 476

Voges, W., Aschenbach, B., Boller, T., et al. 1999, A\&A, 349, 389

Wambsganss, J., Bode, P., \& Ostriker, J. P. 2004, ApJ, 606, L93 
Appendix A: Cluster images

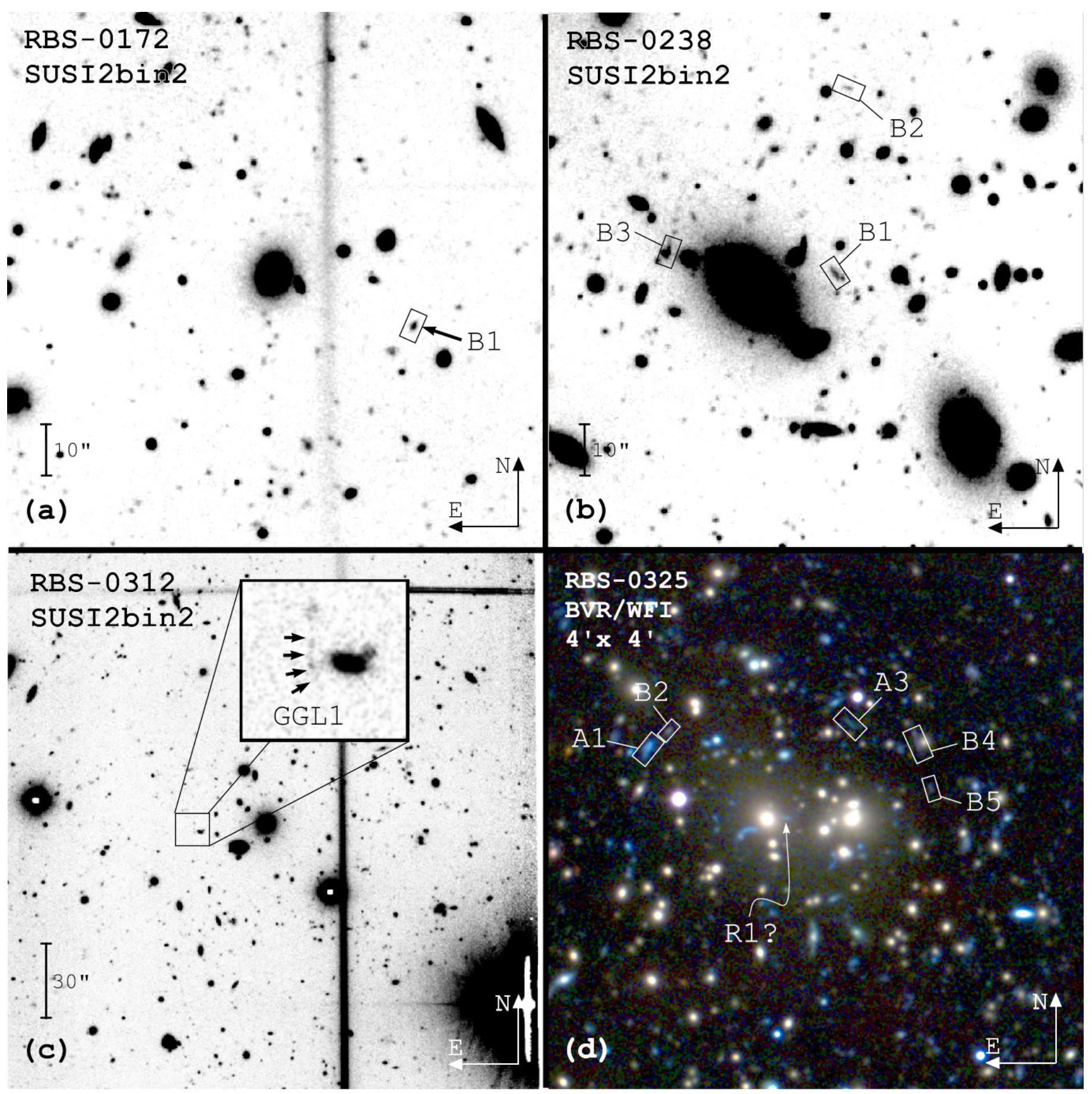

Fig. A.1. Images of all clusters with arc candidates ( $R$-band filter except the three colour composite). The possible strong lensing features are marked by boxes, labelled with "A", and "B" (see Sect.3.1). "SUSI2bin1" ("SUSI2bin2") denotes that the $1 \times 1(2 \times 2)$ binning of the SUSI2 camera was used for this exposure. In all images North is up and East to the left. See Sect. 4 for more details. 
W. Kausch et al.: Arc search in a sample of non-Abell clusters

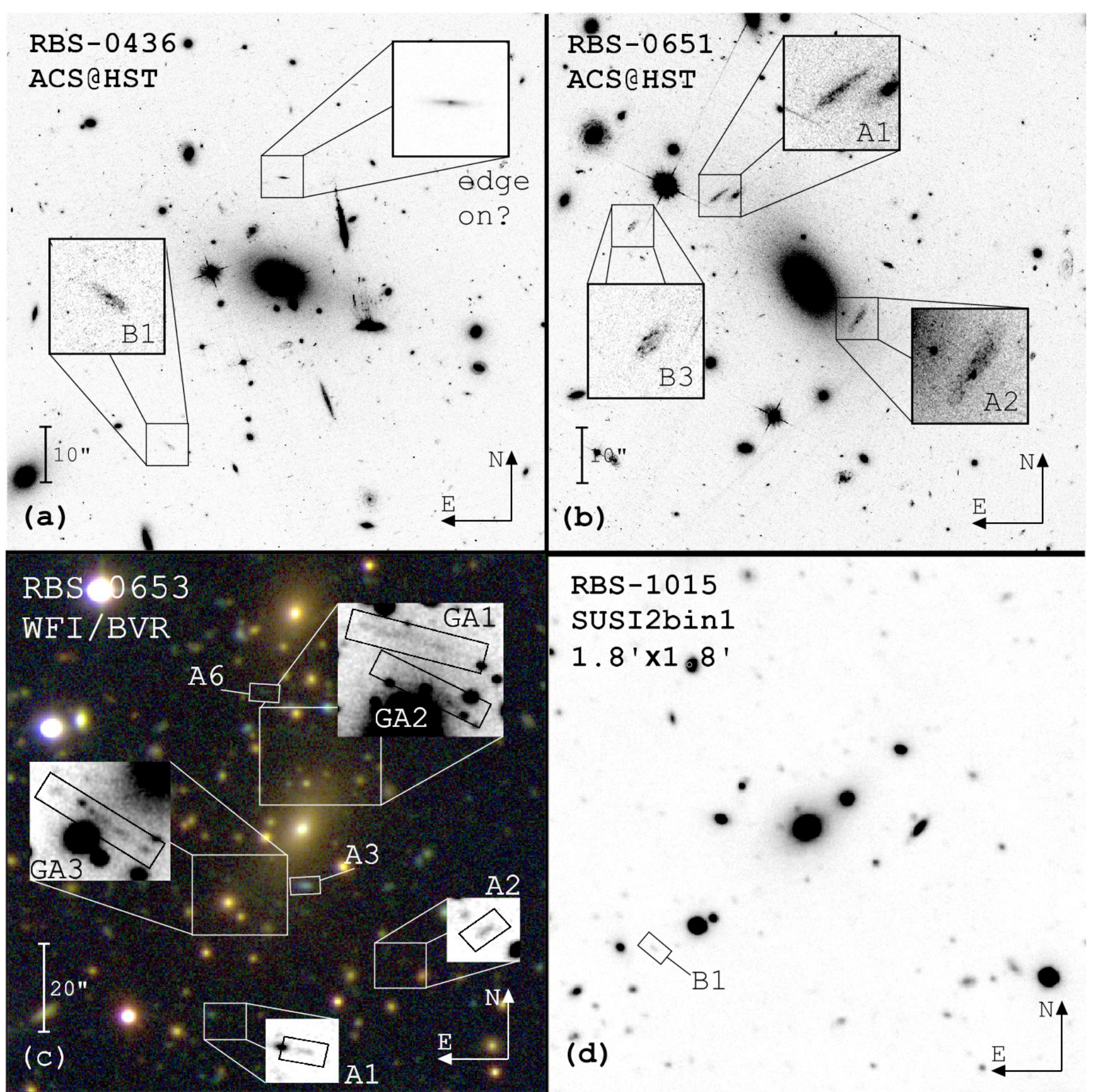

Fig. A.2. Same as Fig. A.1. 


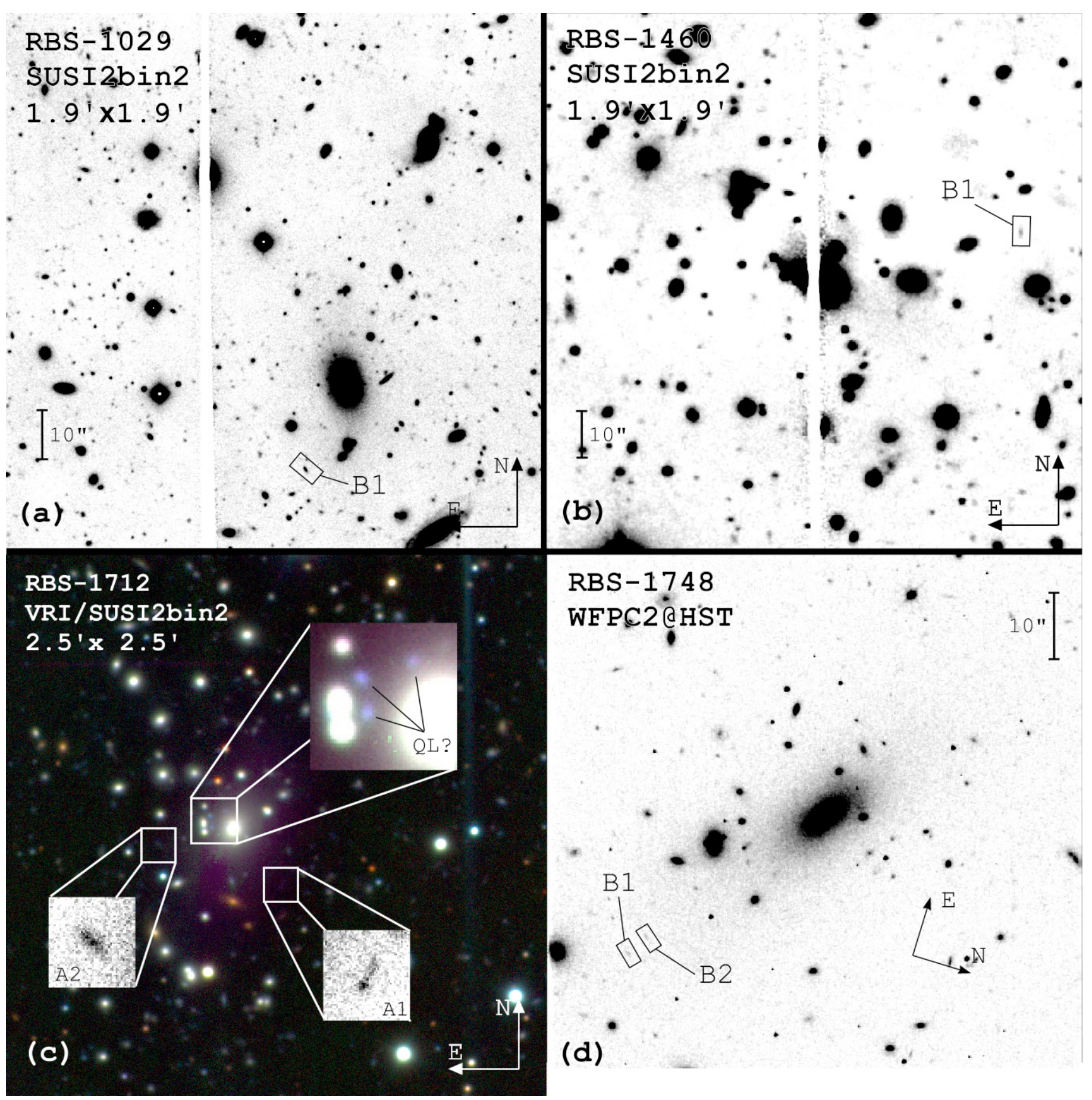

Fig. A.3. same as Fig. A.1. 